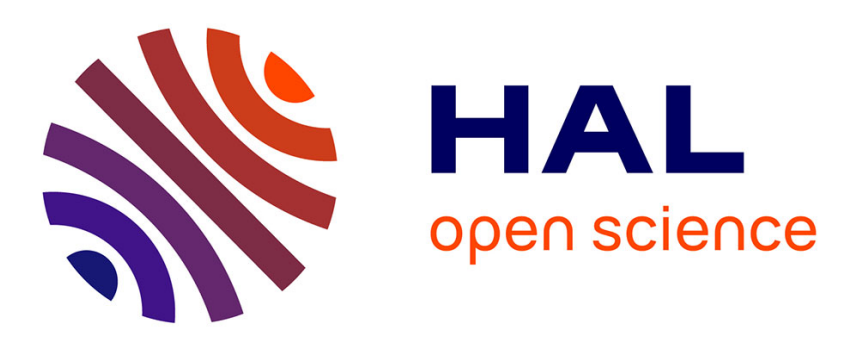

\title{
Adaptation of Classical Evaluation Methods to a Young Audience with Autism Spectrum Disorders
}

\author{
Marine Guffroy
}

\section{To cite this version:}

Marine Guffroy. Adaptation of Classical Evaluation Methods to a Young Audience with Autism Spectrum Disorders. 29ème conférence francophone sur l'Interaction Homme-Machine, AFIHM, Aug 2017, Poitiers, France. 8 p., 10.1145/3132129.3132140 . hal-01578608

\section{HAL Id: hal-01578608 \\ https://hal.science/hal-01578608}

Submitted on 29 Aug 2017

HAL is a multi-disciplinary open access archive for the deposit and dissemination of scientific research documents, whether they are published or not. The documents may come from teaching and research institutions in France or abroad, or from public or private research centers.
L'archive ouverte pluridisciplinaire HAL, est destinée au dépôt et à la diffusion de documents scientifiques de niveau recherche, publiés ou non, émanant des établissements d'enseignement et de recherche français ou étrangers, des laboratoires publics ou privés. 


\title{
Adaptation des méthodes d'évaluation classiques à un jeune public avec Troubles du Spectre Autistique
}

\section{Adaptation of Classical Evaluation Methods to a Young Audience with Autism Spectrum Disorders}

\author{
Marine Guffroy \\ CREN \\ Université du Maine \\ 72000, Le Mans, France \\ marine.guffroy@univ-lemans.fr
}

\begin{abstract}
In order to validate the usability and utility of an application, it is necessary to evaluate it. Many methods exist. The choice of these is defined by the availability of the application and the end users. But other parameters impact this choice as the context of use of the tool as well as the specificities of the public. The methods most commonly used when the application and the users are available, rely predominantly on verbal exchanges, in individual and group interviews. We want to evaluate an application dedicated to a young audience with ASD (Autism Spectrum Disorders). Apart from this audience, there are difficulties in the areas of communication and social interactions. Therefore, how can we evaluate an application dedicated to this particular audience while involving it? We propose an adaptation of classical evaluation methods based on the environment and the human environment of the users.
\end{abstract}

\section{CCS CONCEPTS}

- Human-centered computing $\rightarrow$ User models

\section{KEYWORDS}

Evaluation, specific user, ASD, adaptability

\section{RÉSUMÉ}

Afin de valider l'utilisabilité et l'utilité d'une application, il est nécessaire de l'évaluer. De nombreuses méthodes existent. Le choix de celles-ci est défini par la disponibilité de l'application et celle des utilisateurs finaux. Mais d'autres paramètres entrent en jeu dans ce choix comme le contexte d'utilisation de l'outil ainsi que les particularités du public. Les méthodes

(c) ACM, 2017. This is the author's version of the work. It is posted here by permission of ACM for your personal use. Not for redistribution.

The definitive version was published in Actes de la 29ème conférence francophone sur l'Interaction Homme- Machine, IHM'17, August 28-September 1, 2017, Poitiers, France

https://doi.org/10.1145/3132129.3132140 les plus communément utilisées lorsque l'application et les utilisateurs sont disponibles, reposent majoritairement sur des échanges verbaux, en entretiens individuels comme en groupe. Nous souhaitons évaluer une application dédiée à un jeune public avec TSA (Troubles du Spectre Autistique). Or ce public présente, entre autres, des difficultés dans les domaines de la communication et des interactions sociales. Dès lors, comment évaluer une application dédiée à ce public particulier tout en le faisant participer ? Nous proposons une adaptation des méthodes d'évaluation classiques en nous appuyant sur l'environnement et l'entourage humain des utilisateurs.

\section{MOTS-CLEFS}

Évaluation, public spécifique, TSA, adaptabilité

\section{INTRODUCTION}

Qu'il soit numérique ou non, un outil est utile et utilisable seulement s'il a été bien conçu. S'il ne l'est pas, l'utilisateur ne s'en servira pas. La conception de l'artefact impacte dans tous les cas son cycle de vie. Afin de vérifier que l'outil correspond aux besoins et attentes de l'utilisateur, il est nécessaire de l'évaluer.

Un ensemble de méthodes est disponible afin de réaliser l'évaluation d'un outil [1]. Il existe de nombreuses classifications de ces méthodes [2,3]. Le choix des méthodes est défini par le type de l'outil, mais également par les disponibilités ou non de l'outil dans sa version finale, et des utilisateurs finaux. Les spécificités de ces derniers peuvent également impacter le choix de ces méthodes ainsi que le déroulement de la phase d'évaluation. C'est le cas du public ciblé dans cet article. Évaluer un outil dédié à un jeune public avec TSA (Troubles du Spectre Autistique) impacte le choix des méthodes d'évaluation mais également l'ensemble du déroulement de la phase d'évaluation.

Dans la deuxième partie de ce papier, nous nous intéresserons aux particularités de ce public puis nous mettrons en évidence l'incidence de ces particularités sur le choix des méthodes. Dans une quatrième partie, nous proposerons une méthode d'évaluation adaptée. Cette dernière a fait l'objet d'une étude de cas en situation réelle que nous présenterons en cinquième partie. 


\section{UN JEUNE PUBLIC AVEC TSA}

\subsection{Particularités de ce public}

Les troubles $\mathrm{du}$ spectre autistique sont communément présentés sous la forme de triade (Figure 1) à laquelle s'ajoute un ensemble d'autres troubles comme par exemple des hyper ou hypo sensibilités au niveau des cinq sens, ou encore des difficultés au niveau de la motricité fine.

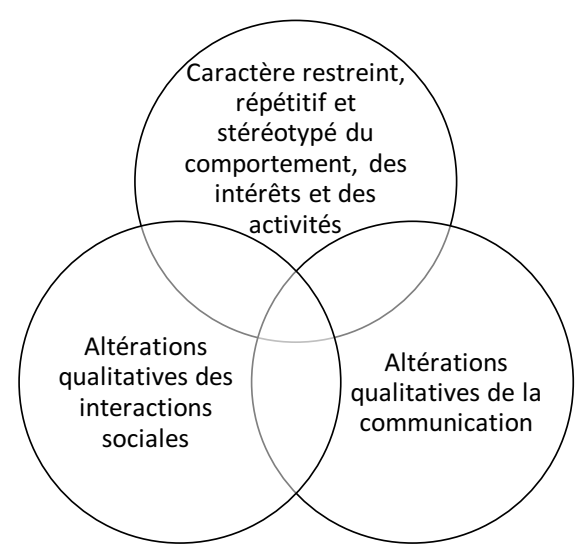

Figure 1 : La triade autistique

Un enfant avec TSA présente une partie plus ou moins importante de ces troubles, c'est pour cela qu'il y a de nombreuses formes d'autisme allant de l'autisme de bas niveau à l'autisme de haut niveau. La classification de ces troubles varie en fonction des pays et de leurs classifications (DSM-V pour les États-Unis [4], CTFMEA pour la France [5], CIM-10 par l'OMS pour la classification internationale [6]). Ce public est très hétérogène dans ses difficultés et donc dans ses besoins.

Il n'y a pas de traitement capable de guérir de l'autisme. Cependant il est couramment recommandé que la prise en charge repose sur trois grands volets :

- éducatif, avec pour objectif l'autonomie de la personne : par les familles ;

- pédagogique, avec pour objectif les apprentissages : par l'Éducation Nationale ;

- thérapeutique, avec pour objectif la santé mentale et physique de la personne : par le personnel de santé.

Les enfants avec TSA sont donc accompagnés au quotidien par différentes personnes.

Du côté informatique, les enfants avec TSA montrent un intérêt grandissant pour les nouvelles technologies. En effet, l'informatique présente une relation stable, patiente et prévisible [7, 8] et offre de nombreux stimuli [9]. L'apparition du tactile a facilité l'interaction, permettant une manipulation du bout des doigts alors que l'utilisation de la souris était parfois compliquée. Des études ont montré que l'utilisation d'applications sur tablette avec ce public améliore la communication [10], ou encore encourage les interactions sociales et le développement cognitif [11]. On observe aujourd'hui une multiplication des applications dédiées à ce public. Mais celles-ci correspondent-elles aux besoins des enfants avec TSA ?

\subsection{Participation de ce public dans les phases de conception et d'évaluation}

Frauenberg et al. [12-14] ont expérimenté la participation des enfants avec TSA dans la conception d'application. Ils notent que différents aspects de l'autisme doivent être pris en compte dans la démarche de conception :

- l'aspect relationnel : une relation solide et durable avec les participants permet un travail participatif prospère. La confiance mutuelle entre tous les acteurs est indispensable pour mener des activités de conception. Cela nécessite du temps et de l'empathie.

- la communication : les activités de conception impliquent une certaine forme de communication entre les concepteurs et les enfants. Cet exercice peut déjà être compliqué pour un adulte ordinaire ; il est d'autant plus difficile pour des enfants TED (Troubles Envahissants du Développement). Il est nécessaire de proposer des aides à la communication adaptées à leurs capacités et besoins particuliers, comme par exemple l'utilisation de pictogrammes.

- la créativité : La conception participative offre une opportunité de puiser dans le potentiel créatif des enfants. Le travail avec des enfants TED nécessite un équilibrage minutieux des possibilités de créativité grâce au soutien des encadrants.

- la traduction : Les productions des enfants ne peuvent pas être utilisées en l'état. Elles doivent être interprétées et traduites pour devenir utilisables. Ce travail peut être particulièrement difficile car l'entrée peut paraître floue et irrationnelle pour l'adulte.

Les auteurs concluent en précisant que la conception pour les enfants avec TSA peut être lourde et qu'il est nécessaire d'alléger l'exercice. Ils insistent sur le fait que les enfants avec TSA ne doivent pas être définis seulement via leurs handicaps mais qu'il faut garder à l'esprit que ce sont des enfants.

Benton et al. arrivent aux mêmes conclusions dans leur étude mais précisent qu'il est nécessaire d'avoir un adulte pour chaque enfant. L'adulte agit ainsi comme médiateur entre l'enfant et l'équipe de projet [15].

Faire participer les enfants avec TSA à la phase d'évaluation d'un outil qui leur est dédié semble donc être possible mais en adaptant les méthodes et le déroulement des séances de travail.

\section{APPLICABILITÉ DES MÉTHODES D'ÉVALUATION CLASSIQUES}

\subsection{Méthodes et techniques d'évaluation disponibles}

Il existe un grand nombre de méthodes et de techniques permettant de mener à bien l'évaluation d'une application interactive. Cependant, ces méthodes ne correspondent pas toujours aux caractéristiques des publics concernés par l'outil. Des paramètres tels que l'âge, les incapacités physiques ou les spécificités relationnelles sont à prendre en compte. L'étude de chaque personnalité offre un éventail de profils particuliers à prendre en considération. L'Universal Access Handbook [16] a répertorié différentes méthodes et techniques potentiellement 
utiles et exploitables en phases de conception et d'évaluation des logiciels. L'étude de leur applicabilité en fonction des profils des utilisateurs a produit une typologie se basant sur l'âge (enfant/personnes âgées) et les incapacités et/ou difficultés rencontrées par ces publics (motricité, vue, audition, cognition, communication). Dans chacun des cas, les méthodes et techniques sont définies comme «appropriées», c'est-à-dire qu'elles peuvent être appliquées dans l'état, comme «nécessitant des modifications ou des ajustements», ou encore comme «non recommandées». Le tableau 1 présente une partie de cette classification en ne retenant que deux paramètres, l'âge (enfant) et les difficultés de cognition/communication, qui correspondent à des facettes caractéristiques de notre public.

Une première hypothèse concernant l'applicabilité des méthodes au jeune public avec TSA, est qu'un croisement des deux paramètres discriminants (enfant et difficultés de communication) peut suffire. Nous complétons le tableau 1 en y ajoutant une colonne, résultat du croisement des deux paramètres discriminants retenus. Cependant nous verrons dans la suite du papier que la réalité est tout autre.

Tableau 1: Tableau récapitulatif de l'applicabilité des méthodes et techniques de conception et d'évaluation en fonction du type de public.

\begin{tabular}{|c|c|c|c|}
\hline & Incapacités/ difficultés & Enfant avec TSA & Age \\
\hline Méthodes et techniques & $\begin{array}{l}\text { Cognitive / } \\
\text { communication }\end{array}$ & $\begin{array}{l}\text { (croisement des colonnes } \\
\text { « Cognitive/communication » } \\
\text { et «Enfant » }\end{array}$ & Enfant \\
\hline Brainstorming & $\mathbf{\square}$ & $\mathbf{\square}$ & $\mathbf{\square}$ \\
\hline Observation directe & $\checkmark$ & $\checkmark$ & $\checkmark$ \\
\hline Agendas d'activités & $\overline{\mathbf{a}}$ & 口 & $\overline{\mathbf{a}}$ \\
\hline Enquêtes et questionnaires & 区 & 凶ा $\rightarrow$ 凶 & $\mathbf{\square}$ \\
\hline Entretiens & 凶 & 凶ा $\rightarrow$ 凶 & $\mathbf{\square}$ \\
\hline Groupes de discussions & 区 & 凶ा $\rightarrow$ 凶 & $\overline{\mathbf{a}}$ \\
\hline Modélisation empathique & 凶 & 凶 & 区 \\
\hline Essais de l'utilisateur & $\mathbf{\square}$ & $\overline{\mathbf{a}}$ & $\mathbf{\square}$ \\
\hline Scénarios, storyboards et personas & $\checkmark$ & $\checkmark$ & $\checkmark$ \\
\hline Prototype & $\checkmark$ & $\checkmark$ & $\checkmark$ \\
\hline Conception coopérative participative & $\mathbf{\square}$ & $\mathbf{\square}$ & $\mathbf{\square}$ \\
\hline
\end{tabular}

$\checkmark$ Méthode ou technique appropriée

- Nécessite des modifications et ajustements

Мéthode ou technique non recommandée

Source: Summary of User Requirements Elicitation Methods, The Universal Access Handbook (15-10)

\subsection{Condition d'applicabilité des méthodes et techniques d'évaluation}

Certaines de ces méthodes et techniques ne sont généralement pas utilisées lors de la phase d'évaluation (scénarios, storyboard, personas, agendas d'activités), nous ne nous y intéresserons donc pas ici.

La littérature sur le public avec TSA est importante. Cependant pour une personne non initiée, comme c'est le cas des membres de l'équipe de la maîtrise d'ouvrage (MOA), porteuse du besoin des utilisateurs, il est impossible de comprendre ou de simuler les particularités propres à ce public. C'est pour cette raison que la modélisation empathique, qui consiste à se mettre à la place de l'utilisateur en simulant ses difficultés (par exemple, utiliser des lunettes déformantes pour simuler un problème de vue) [17] est non envisageable.

Les méthodes reposant sur la communication sont elles aussi difficilement applicables. Il est cependant possible de s'appuyer sur une tierce personne afin d'aider l'enfant à communiquer [15] mais cela demande une adaptation des sessions d'échanges. Toutefois, l'information fournie par l'enfant étant traduite par un médiateur, celle-ci peut être dénaturée.

Un prototype est une représentation concrète de tout ou partie d'un système interactif qui peut être utilisé par les utilisateurs finaux. Le public avec TSA ayant des difficultés d'abstraction et de généralisation, la possibilité de manipulation concrète de l'outil est donc indiquée pour lui. Il est nécessaire de lui présenter un prototype abouti plutôt qu'une maquette de type papier.

L'observation directe consiste à observer l'utilisateur manipuler l'outil à évaluer dans son contexte naturel d'utilisation. Davies et son équipe [18] ont montré que cette méthode, ne reposant pas sur la communication, peut être mise en place avec ce public. Cependant, le cadre de vie d'un enfant avec TSA est borné dans le temps et dans l'espace. Il n'est pas envisageable d'intervenir dans ce cadre sans y avoir été préalablement invité et avoir développé un climat de confiance. Vivian Hill et son équipe [19] précisent que lorsque les utilisateurs sont des enfants avec TSA, il est nécessaire que les personnes extérieures (chargées de l'observation) rencontrent préalablement les encadrants mais également les enfants, lors d'activités non structurées.

Les essais utilisateurs permettent aux utilisateurs finaux d'essayer le produit (complet ou non) dans un cadre contrôlé ou expérimental via un ensemble de tâches à accomplir. Dans le cas d'un jeune public avec TSA, les essais utilisateurs sont nécessairement organisés dans l'environnement standard dans lequel il trouve ses repères. Les données issues de moyens de capture tels que l'eye tracking ou le relevé de manipulation de la souris ne sont pas exploitables. Les études montrent que le 
regard ne reste pas fixe [20] et que les gestes stéréotypés des enfants empêchent toute exploitation des données récoltées.

L'implication des enfants avec TSA dans le processus de conception et d'évaluation d'un outil numérique est recommandé et nécessaire à l'aboutissement du projet. Vis-àvis des difficultés rencontrées par ce public, l'exercice est complexe. Certains contextes imposent parfois que les professionnels ou encadrants soient les seuls à intervenir dans les processus de conception et d'évaluation [21]. Une démarche plus complète est cependant envisageable. Elle nécessite de choisir et d'adapter les méthodes et techniques disponibles.

Notre proposition est de tenir compte du contexte de vie et d'activités du public, de s'appuyer sur l'environnement humain qui l'accompagne quotidiennement. La question est alors de définir les rôles et places de chacun des acteurs, y compris les enfants avec TSA, dans le processus d'évaluation de l'application.

\section{PROPOSITION D’UNE MÉTHODOLOGIE D'ÉVALUATION EN APPUI SUR L'ENVIRONNEMENT ET L'ENTOURAGE HUMAIN DES ENFANTS AVEC TSA}

Notre proposition de méthode d'évaluation adaptée s'appuie sur le contexte humain de proximité des enfants (personnes proches ou intervenants professionnels) et, en conséquence, sur l'adaptation des méthodes et techniques présentées dans la partie précédente.

\subsection{Intégration de l'environnement humain}

Pour participer à l'évaluation, l'enfant avec TSA a besoin de disposer de son environnement habituel, y compris les personnes qui l'accompagnent au quotidien. Ces personnes que nous qualifions d'interlocuteurs médiateurs, du point de vue de la démarche d'évaluation de l'application, sont les personnes proches des utilisateurs finaux, par exemple : membres de la famille, personnels d'accompagnement, professionnels des institutions scolaires et éducatives, professionnels de santé, .... Celles-ci sont expérimentées et légitimes pour contribuer à la compréhension des besoins des utilisateurs dans leur contexte d'activité. Les interlocuteurs médiateurs peuvent d'une part répondre aux différentes questions que la maîtrise d'ouvrage peut se poser sur l'utilisateur, et d'autre part contribuer directement à l'environnement d'activité des utilisateurs cible.

Nous retrouvons ici la notion de " proxy » utilisée par Frauenberger [12] et Hirano [22] pour désigner les interlocuteurs impliqués dans la démarche de conception et d'évaluation. Dans notre cas, les interlocuteurs ne sont pas exclusifs étant donné que les enfants participent également à l'évaluation. Les interlocuteurs médiateurs interviennent comme complément et non en substitution à l'utilisateur final.

\subsection{Prise en compte du contexte relationnel}

Les difficultés de communication des enfants avec TSA n'empêchent pas différentes formes de communication, verbale ou non verbale. La question de la possibilité d'observation et d'analyse des interactions des utilisateurs avec l'outil à évaluer se pose également. Dans le cas d'enfants avec TSA, il n'est pas envisageable d'organiser des ateliers d'expérimentation en dehors de leur environnement habituel : domicile ou institution. Toutefois, rien n'empêche un ingénieur MOA (la Maîtrise d'OuvrAge est l'entité porteuse du besoin des utilisateurs, elle est l'interface entre l'équipe de développement et le public), d'intégrer progressivement ces espaces pour s'y fondre et ainsi permettre à terme une observation directe des usages développés par les enfants avec le produit. Les professionnels du CRA (Centre Ressources Autisme) interrogés indiquent toutefois qu'il ne faut pas que «trop de personnes interviennent », il n'est pas possible de faire intervenir plusieurs personnes dans le cadre de vie de l'enfant avec TSA : un unique représentant de la MOA est en contact avec les enfants. Des activités de " débriefing » courtes sont également envisageables dans ce cadre. Ce type d'intervention nécessite des capacités d'adaptation des différentes parties : accueil et intégration pour les professionnels, implication personnelle et savoir-être pour l'ingénieur MOA. Une fois installé le climat de confiance, il peut également être efficient de travailler directement avec les adultes et professionnels accompagnant les enfants utilisateurs de l'application. Ce principe de croisement des points de vue est développé ci-dessous. Il constitue le principe fondateur d'une méthodologie d'évaluation en appui sur le contexte humain pris dans son ensemble.

\subsection{Combinaison des points de vue}

Dans ce contexte, il est naturel d'étudier et de prendre en compte les différentes situations d'observations et d'usages existantes autour de l'usage de l'application à évaluer, quitte à élargir le cercle des interlocuteurs au-delà des utilisateurs directs de l'application.

Notre proposition de méthodologie d'évaluation d'une application dédiée à un jeune public avec TSA, s'appuie ainsi sur trois catégories d'acteurs déjà présentées :

- les utilisateurs eux-mêmes, qui malgré leurs difficultés de communication, manipulent l'application, et peuvent exprimer, sous différentes formes, leurs avis sur cette utilisation

- les interlocuteurs médiateurs, qui côtoient et connaissent les utilisateurs. Ils sont en mesure d'identifier les profils, les besoins et limites de ces derniers. Ils sont également présents lors de la manipulation et l'utilisation de l'outil par les enfants dans leur contexte ;

- l'ingénieur MOA qui, à condition de s'investir humainement et professionnellement dans le cadre de vie des utilisateurs, peut mener des observations in situ et provoquer certains types d'échanges propices à l'analyse approfondie des interactions qui s'installent entre les enfants et l'application.

Le schéma ci-après (Figure 2) présente les différentes formes d'interactions pouvant être activées entre les différents acteurs. Chacune de ces formes est numérotée et développée dans la suite du texte.

Différentes phases d'observation et d'échanges sont organisées entre les trois types d'acteurs pour obtenir au final une vue d'ensemble assez complète des qualités et défauts de l'application, malgré la difficulté des utilisateurs à exprimer directement leur point de vue à ce sujet. 


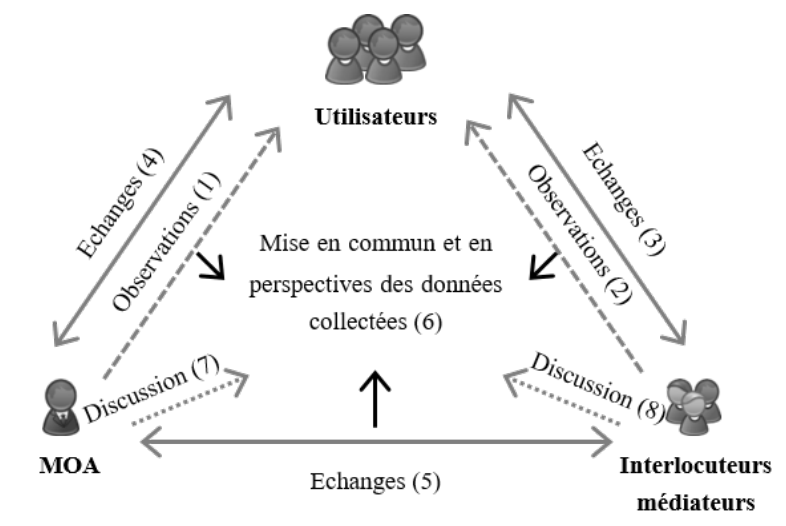

Légende

$A \rightarrow B$ : Échanges verbaux entre A et B

$-A \rightarrow \quad B: A$ observe $B$ en situation

Figure 2 : Modélisation des interactions entre acteurs dans la phase d'évaluation d'une application interactive en contexte spécifique

\section{Phases (1 et 2) d'observation des utilisateurs}

L'activité est observée au sens de Vygotsky [23], c'est-à-dire un sujet, l'enfant, utilisant un artefact, l'outil numérique, en vue d'atteindre un but, réaliser la ou les tâches proposées par l'application. Cependant, Engeström considère que l'activité est inscrite dans un contexte [24], décrit par une communauté régie par des règles où chacun a une place précisément définie. L'intervenant MOA, suite à la phase de mise en confiance, est considéré comme faisant partie de cette communauté, au même titre que les interlocuteurs médiateurs, chacun ayant un rôle défini. Deux types d'acteurs interviennent donc potentiellement comme observateurs des relations entre le sujet et l'objet. N'ayant pas les mêmes objectifs, les mêmes grilles de lecture, ni même la même implication dans la communauté, la MOA et les interlocuteurs médiateurs n'observent finalement pas les mêmes choses pour une même activité de l'enfant.

Observations par la MOA (1). Du point de vue de l'ingénieur MOA, l'observation porte essentiellement sur l'usage de l'outil dans son contexte d'utilisation, généralement à l'occasion de sessions dédiées. Il peut ainsi noter quand, pourquoi et comment l'outil est utilisé, y compris en situation critique, et détailler les interactions que les utilisateurs développent avec leur outil, et en conséquence avec leur environnement. Pour ce type d'observation menée régulièrement in situ par la MOA, le protocole peut finalement être relativement précis, détaillé, et se rapprocher des situations d'expérimentation en laboratoire où sont précisément définis le lieu, le moment, la durée et le scénario de la tâche visant à tester l'application. Les différents utilisateurs du panel ont alors les mêmes tâches à accomplir, dans le même cadre et dans des conditions identiques. Un tel protocole permet de comparer par la suite les informations recueillies ; on observe par exemple que, pour arriver à un même résultat, les utilisateurs ne font pas les mêmes manipulations.

Observations par les interlocuteurs médiateurs (2). Les interlocuteurs médiateurs sont par définition présents en continu lorsque les utilisateurs manipulent l'outil. Ils ont la possibilité d'observer les utilisateurs à tout moment, sans que ces derniers en aient conscience. Ils peuvent ainsi observer les usages de l'outil en contexte, et détecter les changements de comportement qui peuvent apparaître. Il est nécessaire de définir les modalités de collecte des informations considérées intéressantes par les observateurs, via un cahier de liaison par exemple. Accessible à tout moment, celui-ci présente peu de contraintes et beaucoup d'avantages : il est transportable, accessible à tous, et indépendant d'une quelconque source d'énergie. Il peut aussi être envisagé de filmer ces séances si le contexte s'y prête et si les utilisateurs le permettent.

\section{Phases (3-4-5) d'échanges entre les acteurs}

Les interlocuteurs médiateurs interviennent au quotidien comme accompagnateurs auprès des utilisateurs. Les échanges qui sont verbaux ou non (expression faciale, posture) (3) sont dans ces conditions souvent informels. Si les utilisateurs rencontrent des difficultés avec l'outil, ils viennent « naturellement » en parler à leur entourage habituel. Les interlocuteurs médiateurs ont alors un rôle à jouer en termes de collecte des sujets abordés lors de ces échanges avec les utilisateurs, sous forme de transcription de déclaration des utilisateurs dans le cahier de liaisons par exemple.

Plus occasionnellement, les utilisateurs peuvent être amenés à discuter avec la MOA (4), lors des sessions d'observation, en décrivant ce qu'ils sont en train de faire par exemple, ou de manière plus informelle, en signalant les difficultés qu'ils ont rencontrées depuis la dernière séance. Dans ces situations, des supports à la communication, tels que les pictogrammes, peuvent alors être utilisés pour échanger avec les utilisateurs.

Les échanges entre les interlocuteurs médiateurs et la MOA (5) ont lieu à la fois en amont et en aval des interventions de la MOA, afin de préciser le protocole à suivre et de mieux comprendre «ce qui se passe » pendant les sessions de travail et au quotidien. Ces échanges permettent également à la MOA d'accompagner les interlocuteurs médiateurs dans leur rôle et dans la compréhension de l'outil.

\section{Analyse croisée des données récoltées (6-7-8)}

En alternance avec les phases d'observation et d'échanges in situ, des réunions de travail organisées entre la MOA et les interlocuteurs médiateurs (6) permettent de répondre aux objectifs de l'évaluation : vérifier l'accessibilité et l'utilisabilité de l'application numérique. L'analyse croisée des données disponibles (notes d'observation, vidéos, cahier de liaison, ...) permet de répondre aux questions portant à la fois sur les fonctionnalités de l'outil, sur son ergonomie (visuelle en particulier), et sur le comportement des utilisateurs vis-à-vis de l'application. Chaque contributeur apporte son expertise : la MOA identifie les informations utiles à l'évaluation de l'application (7) tandis que les interlocuteurs médiateurs replacent ces informations dans le contexte de l'expérimentation (particularités et besoin du public) (8).

Cette méthodologie d'évaluation d'application interactive dédiée à un public spécifique permet potentiellement de répondre aux objectifs de l'évaluation tout en tenant compte des spécificités du public cible et de son environnement quotidien. 


\section{5 ÉTUDE DE CAS DE LA MÉTHODOLOGIE D'ÉVALUATION PROPOSÉE EN SITUATION RÉELLE}

Nous avons mis à l'essai notre proposition de méthodologie en évaluant l'application çATED (aide à l'autonomie pour les enfants présentant des TED). Cette évaluation a eu lieu d'avril 2014 à juin 2015 dans une ULIS TED (Unité Localisée pour l'Inclusion Scolaire pour enfants avec Troubles Envahissants du Développement).

\subsection{Cadre de l'étude de cas}

L'application que nous avons évaluée pour mettre à l'essai notre méthodologie d'évaluation est l'application çATED

(Figure 3). Celle-ci propose une aide à l'autonomisation grâce à un emploi du temps numérique paramétrable, s'appuyant sur les outils classiquement utilisés par les enfants avec TSA, c'est-à-dire les pictogrammes et le time timer (

Figure 4).

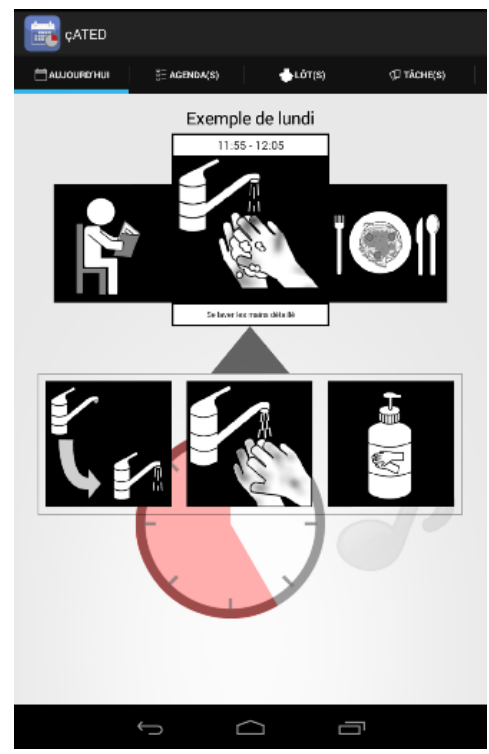

Figure 3 : Écran principal de l'application çATED montrant les tâches précédentes, courante et suivante, avec pour la tâche courante un timer et une liste de sous tâches associées

Les enfants de l'ULIS TED qui ont participé à l'évaluation de l'application çATED avaient entre 6 à 11 ans, et étaient encadrés par une institutrice et deux AVS (Auxiliaire de Vie Scolaire). L'évaluation de la version initiale de l'application a eu lieu d'avril 2014 à mars 2015 (sur 36 semaines au total), le développement d'août 2014 à mars 2015, l'évaluation de la nouvelle version d'avril à juin 2015 (sur 12 semaines). Les élèves des ULIS TED présentent généralement des profils relativement proches. Sur les cinq enfants de l'étude, un était non verbal tandis que les quatre autres arrivaient à échanger verbalement. Tous rencontraient des difficultés plus ou moins importantes au niveau des interactions sociales.

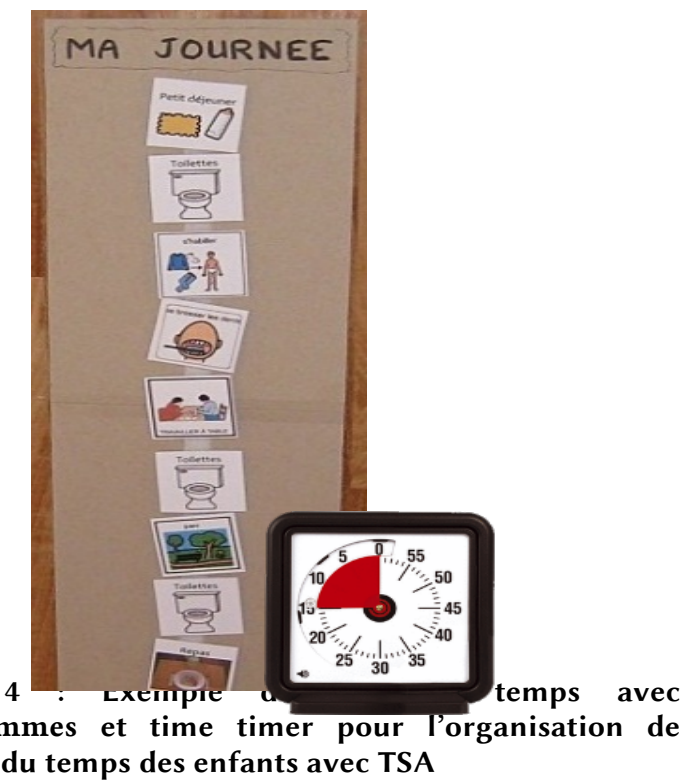

pictogrammes et time timer pour Torganisation de l'emploi du temps des enfants avec TSA

\subsection{Déroulement de l'étude de cas}

Suite aux conseils de l'équipe pédagogique et du CRA (Centre de Ressources Autisme), l'intervenante MOA a tout d'abord réalisé une phase d'immersion et de mise en confiance pendant deux mois, à raison d'une demi-journée par semaine. Pendant cette phase elle participait à l'ensemble des activités de la classe et en co-encadrait certaines. Au terme de cette étape, les enfants étaient à l'aise avec l'intervenante et agissaient en classe comme à leur habitude sans modification du comportement. Des essais d'enregistrement vidéo ont également eu lieu mais ces derniers n'étaient pas concluants. Les enfants étant perturbés par l'usage du matériel d'enregistrement, il a été convenu entre l'intervenante et l'équipe pédagogique que celui-ci ne serait donc pas utilisé dans la suite de l'évaluation.

Des ateliers de travail ont ensuite été organisés afin de permettre à l'intervenante MOA d'observer les enfants manipuler l'application et d'échanger avec eux. Un atelier était encadré par l'intervenante MOA avec un enfant, sur la tablette de ce dernier, au calme dans un coin de la classe ou dans une des salles annexes (laissé au choix de l'enfant), pendant environ 20 minutes (durée moyenne des autres activités de la classe), durant lesquelles l'enfant réalisait son emploi du temps sur sa tablette (1). Des échanges occasionnels avec les élèves (4) pouvaient avoir également lieu en dehors des ateliers, les enfants décrivant alors les problèmes rencontrés au quotidien sur l'application. L'intervenante MOA prenait en notes l'ensemble de ses observations et échanges avec les enfants : observations sur les erreurs de manipulation, la fatigue et la concentration de l'enfant, retranscriptions de certains échanges, avancement dans l'activité. Durant le restant de la semaine seule l'équipe pédagogique observait (2) et échangeait (3) avec les enfants sur l'application, toutes les informations recueillies pendant ces exercices étaient consignées dans le cahier de liaison. Ces informations étaient de différents types : problèmes rencontrés et solutions trouvées, idées de mise en page (placement de composants, couleurs des éléments), 
observations sur l'appropriation des tablettes et de l'application par les élèves.

Les échanges entre la MOA et l'équipe pédagogique (5) avaient lieu chaque semaine en amont et en aval des activités. L'équipe pédagogique s'est rapidement investie, posant des questions et notant quotidiennement ce qu'elle observait. À la fin des sessions de travail, la MOA et l'équipe pédagogique ont mis en commun leurs informations (6) et ont repris ces dernières une à une afin de les présenter et de les commenter (7 et 8). Chacune apportant son expertise, la MOA, l'expertise informatique et l'équipe pédagogique l'expertise sur le public. La MOA a ensuite rédigé le cahier d'exigences de la nouvelle version de l'application çATED en vue de son développement.

\subsection{Bilan de l'étude de cas de la méthodologie d'évaluation proposée}

La phase de mise en confiance a démontré sa nécessité. Il a en effet fallu en moyenne deux séances pour que chaque enfant soit à l'aise avec l'intervenante, et se comporte avec elle comme avec l'équipe pédagogique, sans crainte ni blocage.

L'équipe pédagogique et les enfants se sont fortement investis, les enfants allant au-delà de ce qui leur était proposé au départ (comme par exemple participer volontairement à la rédaction du mode d'emploi de l'application sur leur temps de loisir).

Lors de l'analyse croisée des données récoltées, il est apparu que les types d'informations recueillies sont fortement liés à la source et qu'il n'y a que peu de redondance d'information. Les retours des enfants portaient principalement sur le visuel de l'application (esthétisme, placement et taille des composants) critiquant parfois en se référant aux visuels auxquels ils étaient habitués avec d'autres outils. L'équipe pédagogique a fourni des informations sur la charge de travail, l'homogénéité et la cohérence de la tâche, argumentant ses remarques des connaissances qu'elle a du public, comme par exemple la fatigue que les enfants peuvent avoir à réaliser ce type d'activité ou encore leurs besoins d'avoir des actions découpées en tâches précises et cohérentes. La MOA, quant à elle, a apporté des informations sur le guidage et la gestion des erreurs ; les difficultés motrices des enfants leur faisant faire des actions non désirées sur l'application, ils changeaient d'écran sans être averti, perdant parfois le travail en cours, et sans comprendre pourquoi ils étaient sur un nouvel écran. La MOA et l'équipe de développement ont décidé de refondre complétement l'application en proposant une nouvelle charte graphique, une nouvelle gestion des onglets et l'ajout de nouvelles fonctionnalités.

La nouvelle version de l'application çATED a ensuite été évaluée dans la même classe en suivant le même protocole (mêmes acteurs, format de sessions de travail et de réunions). La seconde évaluation s'est déroulée plus rapidement, la phase de mise en confiance n'étant pas nécessaire et les enfants connaissant déjà l'application. Les retours ont été positifs : toutes les modifications et ajouts de fonctionnalités ont répondu aux problèmes et besoins identifiés lors de l'évaluation de la version précédente de l'application. Ce résultat nous permet de valider la complétude des informations recueillies lors de la première évaluation.
Entre les deux versions de l'application, l'équipe pédagogique et l'intervenante MOA ont observé une diminution du temps de saisie. Par exemple, pour un nombre de tâches à créer équivalant, les enfants n'ont eu besoin que d'une séance de 20 minutes pour toutes les saisir contre en moyenne deux séances avec l'ancienne version. L'institutrice a également noté qu'elle observait moins de fatigue et de frustration avec la nouvelle version, précisant que cela était dû à la réduction significative du nombre d'erreurs de manipulation.

Cette mise à l'essai de la méthode d'évaluation proposée nous a permis de vérifier la légitimité de la place et rôle de chacun des trois types d'acteurs. Les informations recueillies par chacun se complètent et permettent un recueil de retour plus complet que si celui-ci avait eu lieu avec uniquement la MOA et l'équipe pédagogique ou encore la MOA et les enfants.

\section{CONCLUSION}

Le public avec TSA est jeune et a, rappelons-le, des difficultés dans les domaines de la communication et des interactions sociales. Une approche classique d'évaluation reposant sur la communication ne pouvant pas être mise en œuvre, il a été nécessaire de trouver une alternative, tout en gardant une place pour l'utilisateur dans le processus d'évaluation. L'objectif est de permettre au public utilisateur de participer activement à l'évaluation de l'application qui lui est proposée, de lui donner la possibilité d'exprimer, sous différentes formes, son avis et ses préconisations sur l'outil. Notre proposition méthodologique s'appuie sur la prise en compte du contexte humain environnant l'utilisateur. Elle combine plusieurs techniques d'évaluation dans le cadre d'un protocole précis et repose sur la contribution de trois types d'acteurs: utilisateurs, interlocuteurs médiateurs et MOA. L'adaptation des techniques permet de conserver la finalité de l'évaluation de logiciel tout en prenant en considération le contexte d'usage réel : les tests utilisateurs sur le prototype sont réalisés in situ avec le public cible, les phases d'analyse des usages sont effectuées avec les interlocuteurs médiateurs sur la base d'observations issues du terrain, la mise en perspective des résultats est effectuée selon les points de vue de chacun.

Sur ce dernier point, on peut souligner que dans le cas de l'évaluation de l'application çATED, les enfants se sont positionnés sur l'esthétique et le placement des composants d'interface ; que les professionnels accompagnateurs se sont particulièrement intéressés à la charge de travail, l'homogénéité et la cohérence des interactions produites avec l'application ; tandis que la MOA s'est concentrée sur la qualité de l'ergonomie en termes de guidage et de gestion des erreurs. Les trois catégories d'acteurs apportent donc des informations complémentaires et peu redondantes. Le retrait de l'un de ces acteurs soustrairait une partie des retours. Nous notons qu'aucun des retours ne concerne les fonctionnalités de l'application çATED, celle-ci répondant aux besoins de gestion du temps des élèves. Nous émettons l'hypothèse que des retours sur les fonctionnalités pourraient être émis à la fois par les utilisateurs qui peuvent juger eux-mêmes de leurs besoins mais également par les interlocuteurs médiateurs, conscients eux-aussi des besoins des utilisateurs. Une nouvelle 
mise à l'essai avec une autre application et un autre panel permettrait de valider cette hypothèse et de confirmer la validité de notre proposition de méthodologie d'évaluation adaptée à un jeune public avec TSA. Une autre question se pose également au niveau de la cohérence et de l'homogénéité des propositions des enfants TSA eux-mêmes et celles des interlocuteurs médiateurs. En effet, il est possible que ces avis diffèrent. Les proches des enfants avec TSA entendent souvent beaucoup des outils proposés, souhaitant des applications proposant énormément de fonctionnalités.

Ces résultats ont été obtenus grâce à une profonde remise en cause des méthodes d'évaluation habituelles, et surtout grâce à un questionnement sur le rôle de chacun dans cette phase d'évaluation qui tient une place centrale dans le cycle de conception d'une application numérique : sans évaluation avec les utilisateurs, il n'est pas possible d'obtenir les retours d'usage nécessaires à son amélioration. Le questionnement collectif a abouti à des adaptations de rôle pour différents acteurs : présence et posture (savoir-être) pour la MOA, temps dédiés à une nouvelle activité pour le public usager, à la fois implication et prise de recul pour les professionnels accompagnateurs. Chacun s'est investi dans cette activité, s'est impliqué dans le processus, s'est senti acteur de l'évaluation. Ce sont là les conditions de concrétisation d'une démarche d'évaluation aboutie d'un logiciel dédié à un public spécifique.

\section{REMERCIEMENTS}

Cette recherche est effectuée dans le cadre du projet « çATEDautisme » qui est mené par une équipe pluridisciplinaire de chercheurs en éducation et en informatique des Universités de Nantes et du Maine, regroupés au sein du Centre de Recherche en Éducation de Nantes (CREN-EA 2661), par les professionnels du Centre de Ressources Autisme des Pays de la Loire (CRA), par une équipe hospitalo-universitaire de l'Université d'Angers et par des ingénieurs de la société SII Ouest Centre Atlantique. Nous tenons à remercier ces partenaires, ainsi que les enfants, l'institutrice et les AVS de l'ULIS, de nous avoir si chaleureusement accueillis et de s'être investis dans ce projet.

\section{RÉFÉRENCES}

[1] B. Senach, «Evaluation ergonomique des interfaces homme-machine: une revue de la littérature », INRIA, Rapport Technique, 1990.

[2] H. Ezzedine, A. Trabelsi, C. D. Tran, et C. Kolski, « Critères et méthodes d'évaluation des systèmes interactifs, Application à un poste de régulation dans le domaine des transports », in Ingénierie du transport et des services de mobilité avancés, Hermes Science Publishing Ltd., Paris: S. Hammadi, M. Ksouri, 2012, p. 213-293.

[3] J. Huart, C. Kolski, et C. Bastien, "L'évaluation de documents multimédias, Etat de l'art», in Objectiver l'humain? Volume 1, Qualification, quantification, Hermes, vol. 1, Paris: Merviel S, 2008, p. 211-250.
[4] American Psychiatric Association, DSM-V, American Psychiatric Association. 2013.

[5] R. Misès et al., CFTMEA - Classification Française des Troubles Mentaux de l'Enfant et de l'Adolescent - R2012, EHESP-5ème édition. 2012.

[6] OMS, CIM10 (Classification Internationale des Maladies). 2015.

[7] D. K. C. Murray, « Autism and information technology: therapy with computers. », Autism Learn. - Guide Good Pract., p. 100-115, 1997.

[8] G. Rajendran et P. Mitchell, «Computer mediated interaction in Asperger's syndrome: the Bubble Dialogue program. ", Comput. Educ., vol. $35, n^{\circ} 3$, p. $189-207$, nov. 2000.

[9] A. Bosseler et D. W. Massaro, «Development and evaluation of a computer-animated tutor for vocabulary and language learning in children with autism », f. Autism Dev. Disord., vol. 33, n 6 6, p. 653-672, déc. 2003.

[10] J. P. Hourcade, N. Bullock-Rest, et T. E. Hansen, « Multitouch tablet applications and activities to enhance the social skills of children with autism spectrum disorders », Pers. Ubiquitous Comput., vol. 16, $\mathrm{n}^{\mathrm{o}} 2$, $\mathrm{p}$ 157-168, févr. 2012

[11] R. Muñoz, T. Barcelos, R. Noel, et S. Kreisel, « Development of Software that Supports the Improvement of the Empathy in Children with Autism Spectrum Disorder », in Chilean Computer Science Society (SCCC), 2012 31st International Conference of the, 2012, p. 223-228.

[12] C. Frauenberger, J. Good, et A. Alcorn, « Challenges, opportunities and future perspectives in including children with disabilities in the design of interactive technology ", in Proceedings of the 11th International Conference on Interaction Design and Children, 2012, p. 367-370.

[13] C. Frauenberger, J. Good, et W. Keay-Bright, « Designing technology for children with special needs: bridging perspectives through participatory design », CoDesign, vol. 7, $\mathrm{n}^{\circ}$ 1, p. 1-28, mars 2011.

[14] C. Frauenberger, J. Good, A. Alcorn, et H. Pain, « Supporting the design contributions of children with autism spectrum conditions», in Proceedings of the 11th International Conference on Interaction Design and Children, 2012, p. 134-143.

[15] L. Benton, H. Johnson, E. Ashwin, M. Brosnan, et B. Grawemeyer, « Developing IDEAS: Supporting Children with Autism Within a Participatory Design Team ", in Proceedings of the SIGCHI Conference on Human Factors in Computing Systems, New York, NY, USA, 2012, p. 2599-2608.

[16] M. Antona, S. Ntoa, I. Adami, et C. Stephanidis, « Chapter 15 - User Requirements Elicitation for Universal Access », in The Universal Access Handbook, CRC Press, 2009, p. 15-1-14.

[17] L. A. Pastalan, «Environmental design and adaptation to the visual environment of the elderly », in Aging and Human Visual Function, New York: A.R. Liss, 1982, p. 323-333.

[18] R. Davies, S. Marcella, J. McGrenere, et B. Purves, «The Ethnographically Informed Participatory Design of a PD Application to Support Communication », in Proceedings of the 6th International ACM SIGACCESS Conference on Computers and Accessibility, New York, NY, USA, 2004, p. 153-160.

[19] V. Hill, A. Croydon, S. Greathead, K. Lorcan, R. Yates, et E. Pellicano, «Research methods for children with multiple needs: Developing techniques to facilitate all children and young people to have "a voice" », Educ. Child Psychol., vol. 33, n 3, p. 26-43, sept. 2016.

[20] A. C. Manrique, C. D. Jiménez de Espinoza, et J. Gonzàlez-Mora, « A fast automated diagnosis system for autism spectrum disorders based on eye tracking technology », présenté à THE LANCET Neurology Conference 2016, London (UK), 2016.

[21] G. De Leo et G. Leroy, «Smartphones to Facilitate Communication and Improve Social Skills of Children with Severe Autism Spectrum Disorder: Special Education Teachers As Proxies », in Proceedings of the 7th International Conference on Interaction Design and Children, New York, NY, USA, 2008, p. 45-48.

[22] S. H. Hirano, M. T. Yeganyan, G. Marcu, D. H. Nguyen, L. A. Boyd, et G. R. Hayes, "vSked: Evaluation of a System to Support Classroom Activities for Children with Autism », in Proceedings of the SIGCHI Conference on Human Factors in Computing Systems, 2010, p. 1633-1642.

[23] L. Vygotsky, «The instrumental method in psychology», in The concept of activity in Soviet psychology, 1981, p. 134-143.

[24] Y. Engeström, "Towards an expansive methodology », in Learning by Expanding: An Activity - Theoretical Approach to Developmental Research, Helsinki: Orienta-Konsultit, 1987, p. 247-257. 